\title{
Association of perfectionistic and dependent dysfunctional attitudes with subthreshold depression
}

This article was published in the following Dove Press journal:

Psychology Research and Behavior Management

14 August 2017

Number of times this article has been viewed

\author{
Satoshi Horiuchi' \\ Shuntaro Aoki ${ }^{2,3}$ \\ Koki Takagaki ${ }^{4}$ \\ Fumihito Shoji ${ }^{5}$ \\ 'Faculty of Social Welfare, Iwate \\ Prefectural University, Iwate, Japan; \\ ${ }^{2}$ Graduate School of Psychological \\ Science, Health Sciences University \\ of Hokkaido, Hokkaido, Japan; \\ ${ }^{3}$ Japan Society for the Promotion of \\ Science, Tokyo, Japan; ${ }^{4}$ Department \\ of Psychiatry and Neurosciences, \\ Hiroshima University, Hiroshima, \\ Japan; ${ }^{5}$ Graduate School of Social \\ Welfare, Iwate Prefectural University, \\ Iwate, Japan
}

Background: Dysfunctional attitudes are beliefs and attitudes that induce negative thoughts about the self, others, and the future, leading to depression. Perfectionistic dysfunctional attitudes are beliefs and attitudes about achievement and excessive fear of failure, while dependent dysfunctional attitudes are beliefs and attitudes about dependency on, and approval from, others. Subthreshold depression refers to a depressive state that does not meet the diagnostic criteria for a major depressive episode. This study examined whether the difference in perfectionistic dysfunctional attitudes between college students with subthreshold depression and those with no depression would be greater than that of dependent dysfunctional attitudes.

Methods: Participants were defined as having subthreshold depression if they scored 16 or higher on the Japanese version of the Center for Epidemiologic Studies Depression Scale but did not meet the diagnostic criteria for a major depressive episode, as assessed by the major depressive episode module of the Mini-International Neuropsychiatric Interview. The participants included 21 male and 87 female college students, with a mean age of 20.3 years. Dysfunctional attitudes were evaluated with the Japanese version of the 24-item Dysfunctional Attitude Scale. Results: Of the 108 students, 34 had subthreshold depression while 74 had no depression. The magnitudes of the differences in perfectionistic and dependent dysfunctional attitudes between the students with subthreshold depression and those with no depression were large (Cohen's $d=0.83$ ) and small (Cohen's $d=0.25$ ).

Conclusion: The results of this study provide the first evidence that the difference in perfectionistic dysfunctional attitudes between college students with subthreshold depression and those with no depression is greater than that of dependent dysfunctional attitudes.

Keywords: subthreshold depression, dysfunctional attitudes, dysfunctional attitude scale, perfectionism

\section{Introduction}

Recently, increased attention has been paid to subthreshold depression. ${ }^{1}$ Subthreshold depression has been defined in a variety of ways and has been used in the psychiatric literature interchangeably with minor depression, mild depression, or subclinical depression. ${ }^{1,2}$ This study defines subthreshold depression as a depressive state that does not meet the diagnostic criteria for a major depressive episode. Subthreshold depression has been reported to be highly prevalent, ${ }^{1}$ to increase the risk of being diagnosed with major depressive disorder, ${ }^{3,4}$ and to adversely affect quality of life. ${ }^{4}$ To develop and evaluate cognitive and behavioral interventions, it is important to identify the behavioral and cognitive mechanisms through which subthreshold depression occurs
Correspondence: Satoshi Horiuchi Faculty of Social Welfare, Iwate Prefectural University, 152-52 Sugo, Takizawa-shi, Iwate-ken 020-0693, Japan

Tel +8I 196942000

Fax +81 196942001

Email horiuchi@iwate-pu.ac.jp 
and is maintained. Compared with behavioral factors, ${ }^{5,6}$ information about cognitive factors related to subthreshold depression is limited.

Unfortunately, there are only a few studies on the cognitive factors of subthreshold depression. Among these, Koenig $^{7}$ investigated whether dysfunctional attitudes were associated with minor depression, which is a form of subthreshold depression that occurs in medically ill patients. Dysfunctional attitudes are beliefs and attitudes that can lead to depression by causing negative thoughts about the self, others, and the future. ${ }^{8}$ Koenig $^{7}$ conducted logistic regression analyses to examine whether global dysfunctional attitudes, which were evaluated using the total score of a 15-item version of a dysfunctional attitude scale for medically ill elderly people, ${ }^{9}$ would distinguish between participants with minor depression and healthy counterparts. Dysfunctional attitudes, in general, were not found to be associated with minor depression, suggesting that there is no association with subthreshold depression.

However, Koenig ${ }^{7}$ focused on global dysfunctional attitudes rather than specific dysfunctional attitudes. Although no direct evidence exists for subthreshold depression, it is well known that specific dysfunctional attitudes have a stronger association with depressive symptoms. For example, Eberhart et $\mathrm{al}^{10}$ examined the associations of several dysfunctional attitudes and stress with depressive symptoms in a sample of college students. Among the variety of dysfunctional attitudes, specific dysfunctional attitudes (namely, self-sacrifice dysfunctional attitudes) were associated with depressive symptoms when stress levels were high. Other dysfunctional attitudes did not show such an interaction with stress. In addition, they found that several dysfunctional attitudes, such as encompassing emotional deprivation, mistrust/ abuse, social isolation, defectiveness, failure, and subjugation, generated interpersonal stress, which in turn increased depressive symptoms. These results suggest that focusing on specific rather than global dysfunctional attitudes has the potential to increase our understanding of the occurrence and maintenance of depressive symptoms to a greater extent than global dysfunctional attitudes. It seems necessary to examine specific dysfunctional attitudes that may have an association with subthreshold depression.

Pertinent to this study, some studies have reported that perfectionistic dysfunctional attitudes are more strongly associated with depressive symptoms than dependent dysfunctional attitudes. ${ }^{11,12}$ Perfectionistic dysfunctional attitudes are beliefs and attitudes about achievement and excessive fear of failure, whereas dependent dysfunctional attitudes are beliefs and attitudes about dependency on, and approval from, others. de Graaf et $\mathrm{al}^{12}$ reported that perfectionistic dysfunctional attitudes are more strongly related to depressive symptoms $(r=0.61)$ than are dependent dysfunctional attitudes $(r=0.51)$. The fact that stronger associations of perfectionistic dysfunctional attitudes than dependent dysfunctional attitudes with depressive symptoms have been reported in previous studies ${ }^{10,11}$ does not necessarily indicate that this is true for subthreshold depression. Dysfunctional attitudes are related to major depression, but not to minor depression, ${ }^{7}$ and it is possible that such stronger associations can be found in major depression. It is potentially important to directly test if perfectionistic dysfunctional attitudes are more strongly associated with subthreshold depression than are dependent dysfunctional attitudes. Such an investigation would indicate the necessity of focusing on specific dysfunctional attitudes when examining the cognitive mechanisms through which subthreshold depression occurs and is maintained.

To address this issue, it is first necessary to examine differences in perfectionistic and dependent dysfunctional attitudes between college students with subthreshold depression and those with no depression. Therefore, the purpose of this study was to test the hypothesis that the difference in perfectionistic dysfunctional attitudes between college students with subthreshold depression and those with no depression would be greater than that of dependent dysfunctional attitudes. Finding a greater difference for perfectionistic dysfunctional attitudes will provide the rationale to focus on specific dysfunctional attitudes.

\section{Materials and methods \\ Participants}

This study was conducted in a public college in the Tohoku region, Japan. The authors chose the college because of its convenience. College students who were not receiving medical care at a hospital or clinic, were not receiving counseling, and did not meet the criteria for a major depressive episode were invited to participate in this study. College students were chosen as the participants in this study, whereas previous studies have focused on medically ill patients ${ }^{7}$ and general populations. ${ }^{10,12}$ Compared to these populations, a sample of college students is more homogeneous, and the results may be less influenced by variations in demographic (eg, age) and clinical variables (comorbidity, physical health). A total of 178 students were invited after lectures and 94 were invited privately. One of the authors $(\mathrm{SH})$ is a faculty member of the college. Another author (FS) recruited participants in order to not pressure the students into participating in this study. 
Of the 110 students who agreed to participate, one was under medical care and one provided incomplete data, leaving 108 in the study ( 21 men and 87 women). They ranged in age from 18 to 22 years (mean 20.3 years \pm 1.18 ). Among the participants, $10.2 \%$ were freshmen, $34.3 \%$ were sophomores, $21.3 \%$ were juniors, and $34.3 \%$ were seniors. A power analysis was not conducted to determine an adequate sample size due to the lack of published data. The number of participants was determined based on the authors' research budget.

\section{Measurement tools}

\section{Major depressive episode}

The presence of a major depressive episode was assessed using the major depressive episode module of the Japanese version of the Mini-International Neuropsychiatric InterviewPlus (MINI). ${ }^{13}$ The MINI, which is a semi-structured interview that lasts for about 10 minutes, was conducted by a masters-level graduate student (FS) or a certified clinical psychologist ( $\mathrm{SH})$. This semi-structured interview has been found to be reliable and valid. ${ }^{13}$ The MINI interview was chosen to screen students with a major depressive episode because it can be easily and quickly administered.

\section{Depressive symptoms}

Levels of depressive symptoms were assessed using the Japanese version of the Center for Epidemiologic Studies Depression Scale (CES-D). ${ }^{14}$ This is a single scale with 20 items that assess the severity of depressive symptoms over the previous week. Each participant was asked to rate each symptom on a 4-point Likert scale ranging from 1 (less than 1 day) to 4 (5-7 days). The scores of all of the items were summed, with higher scores reflecting more severe symptoms. This scale has been found to be reliable and valid. ${ }^{14}$

\section{Dysfunctional attitudes}

Levels of dysfunctional attitudes were assessed using the 24-item Dysfunctional Attitude Scale (DAS-24). ${ }^{15}$ The 24 items comprise three subscales, including achievement, self-control, and dependency. The self-control items have not been found to yield consistent results, while the achievement and dependency items have. ${ }^{12}$ Data from the achievement and dependency subscales were analyzed to test the study hypothesis. The DAS-24 achievement and dependency subscales assess perfectionistic and dependent dysfunctional attitudes with 11 items and 5 items, respectively. Each participant was asked to rate each item on a 7-point Likert scale ranging from 1 (strongly disagree) to 7 (strongly agree). The scores of the achievement and dependency subscales range from 11 to 77 and from 5 to 35, respectively. The scores of all of the items were summed, with higher scores reflecting more dysfunctional attitudes. This scale has been found to be reliable and valid. ${ }^{15}$

\section{Procedure}

This study was approved by the Institutional Review Board of Iwate Prefectural University (no. 143 and 167) and was conducted from January to February, from May to July, and from October to December 2016. The study was not conducted from March to April or from August to September 2016 because it was difficult to collect data in these months due to the academic schedule. The 2015 school year ended at the beginning of February 2016 and the new school year started at the beginning of April 2016. Students were on their vacations during March, August, and September. Each participant completed the questionnaires and the MINI interview. Prior to the study, the purposes and procedures of the study, possible publication of results, the rights of participants (eg, the right to refuse to participate without penalty or to drop out at any time during the study), and incentives were thoroughly explained to each participant, and informed written consent was obtained. The MINI Interview was scheduled according to each participant's availability. They also completed a packet of questionnaires, which they posted into a return box. After completing the MINI interview, a gift card (500 Japanese yen) was given as an incentive.

\section{Statistical analysis}

The significance level was set at less than 5\%. Data analyses except post hoc power analyses were conducted using IBM SPSS Statistics for Windows version 22 (IBM Corp., Armonk, NY, USA). Post hoc power analyses were conducted using $\mathrm{R}$ software (R Foundation for Statistical Computing, Vienna, Austria). The age, male/female ratio, and levels of depressive symptoms and dysfunctional attitudes were then compared between those with subthreshold depression and those with no depression using the $\chi^{2}$ test and $t$-test (Table 1). $\varphi^{2}$ and Cohen's $d$ values were calculated to interpret how large these differences were. According to Cohen (1988), ${ }^{16} \varphi^{2}$ values of $0.01,0.06$, and 0.14 and $d$ values of $0.20,0.50$, and 0.80 , are interpreted as small, medium, and large, respectively. Post hoc power analyses were also conducted. Means with standard deviations and correlations among the studied variables were calculated (Table 2). 
Table I Demographics and levels of depressive symptoms and dysfunctional attitudes in college students with subthreshold depression and those with no depression

\begin{tabular}{|c|c|c|c|c|c|}
\hline Variables & $\begin{array}{l}\text { Students with no } \\
\text { depression } \\
(n=74)\end{array}$ & $\begin{array}{l}\text { Students with subthreshold } \\
\text { depression }(n=34)\end{array}$ & $t$ or $\chi^{2}$ values & $\begin{array}{l}\text { Cohen's } d \text { or } \\
\varphi^{2} \text { values }\end{array}$ & $\begin{array}{l}\text { Post hoc } \\
\text { observed power }\end{array}$ \\
\hline Age, M (SD) & $20.3(1.15)$ & $20.4(1.05)$ & $t(75.7)=-0.37$ & $d=0.09$ & \\
\hline Men, n (\%) & II (I4.9) & $10(29.4)$ & $\chi^{2}(1)=3.15$ & $\varphi^{2}=0.003$ & \\
\hline CES-D, M (SD) & $8.9(3.75)$ & $23.2(5.47)$ & $t(47.8)=-13.83^{\mathrm{a}}$ & $d=3.31$ & \\
\hline DAS-24 achievement subscale & $35.1(7.36)$ & $42.3(11.36)$ & $t(46.2)=-3.39^{\mathrm{a}}$ & $d=0.83$ & 0.98 \\
\hline DAS-24 dependency subscale & $25.9(6.78)$ & $27.5(5.70)$ & $t(106)=-1.19$ & $d=0.25$ & 0.25 \\
\hline
\end{tabular}

Notes: ${ }^{a} p<0.01$.

Abbreviations: CES-D, Center for Epidemiologic Studies Depression Scale; DAS-24, 24-item Dysfunctional Attitude Scale.

Table 2 Means with standard deviations and Pearson correlations among the scores for depression, achievement, and dependency

\begin{tabular}{lllll}
\hline Variables & Mean (SD) & CES-D & DAS-24 A & DAS-24 D \\
\hline I. CES-D & $13.4(7.96)$ & & & \\
2. DAS-24 A & $37.4(9.38)$ & $0.42^{\mathrm{a}}$ & & \\
3. DAS-24 D & $26.4(6.48)$ & 0.13 & $0.40^{\mathrm{a}}$ & \\
\hline
\end{tabular}

Notes: ${ }^{a} p<0.01$.

Abbreviations: CES-D, Center for Epidemiologic Studies Depression Scale; DAS-24 A, 24-item Dysfunctional Attitude Scale Achievement; DAS-24 D, 24-item Dysfunctional Attitude Scale Dependency; SD, standard deviation.

\section{Results}

The results of the MINI and CES-D indicated that, of the 108 students, 34 have subthreshold depression while 74 had no depression. Table 1 presents the comparison of the mean age, male/female ratio, mean levels of depressive symptoms, and the two dysfunctional attitudes between students with subthreshold depression and those with no depression. A series of Student's $t$-tests and $\chi^{2}$ tests indicated that both groups were fairly matched with regard to age $(t[75.7]=-0.37, p=0.73, d=0.09)$, male/female ratio $\left(\chi^{2}[1]=3.15, p=0.08, \varphi^{2}=0.003\right)$, and scores of the dependency subscale $(t[106]=-1.19, p=0.24, d=0.25)$. However, they differed significantly in terms of the scores of the CES-D $(t[47.8]=-13.83, p<0.01, d=3.31)$ and the DAS24 achievement subscale $(t[46.2]=-3.39, p<0.01, d=0.83)$. The magnitudes of the differences in age, male/female ratio, levels of depressive symptoms, achievement, and dependency between the groups were very small, nearly zero, large, large, and small, respectively. Results of post hoc power analyses ( $\mathrm{n} 1=74, \mathrm{n} 2=34, \alpha=0.05$, effect size $=0.25$ or 0.83 ) showed that observed power was 0.98 for the achievement score and 0.25 for the dependency score. Table 2 shows the means with standard deviations and correlations among the studied variables.

\section{Discussion}

The purpose of this study was to test the hypothesis that the difference in perfectionistic dysfunctional attitudes between college students with subthreshold depression and those with no depression would be greater than that of dependent dysfunctional attitudes. The hypothesis was supported. As predicted, the magnitudes of the differences in perfectionistic and dependent dysfunctional attitudes between the participants with subthreshold depression and with no depression were large and small. The observed power estimates of the tests for perfectionistic and dependent dysfunctional attitudes were sufficient and insufficient.

These results are consistent with, and extend, the findings of previous studies ${ }^{11,12}$ showing that perfectionistic dysfunctional attitudes are more strongly related to depressive symptoms. While previous studies have assessed levels of depressive symptoms with scales such as the CES-D or have focused only participants with minor and major depression, this study directly evaluated the presence or absence of subthreshold depression using the CES-D and MINI. By specifically assessing the presence of subthreshold depression, the results of this study provide one of the first lines of evidence that, compared with dependent dysfunctional attitudes, perfectionistic attitudes show greater difference between college students with subthreshold depression and those with no depression. This direct specification of subthreshold depression with MINI is important. Dysfunctional attitudes can be related not to subthreshold depression but to major depression. ${ }^{7}$ Depressive symptoms that are typically measured with self-reported scales such as CES-D can include subthreshold and major depression. Significant associations of dysfunctional attitudes with depressive symptoms do not necessarily indicate that dysfunctional attitudes is associated with subthreshold depression. Therefore, the results of this study provided more robust evidence showing specific associations of perfectionistic dysfunctional attitudes with subthreshold depression.

The association of specific dysfunctional attitudes with subthreshold depression that was found in this study has some implications. The finding supports the proposal of de Graaf et $\mathrm{al}^{12}$ that the use of the DAS's subscales provides more useful information for examining cognitive vulnerability than does its global score. Knowledge of the associations of 
dysfunctional attitudes with depressive symptoms, including major depression and subthreshold depression, have been enhanced by examining specific dysfunctional attitudes. ${ }^{10}$ Global dysfunctional attitudes have been examined in the literature of subthreshold depression. ${ }^{7}$ The findings of this study provide preliminary but clear evidence that perfectionistic dysfunctional attitudes are more strongly associated with the symptoms of subthreshold depression than are dependent dysfunctional attitudes. These results shed new light on the importance of focusing on specific dysfunctional attitudes in order to more accurately clarify the cognitive mechanisms of subthreshold depression in future studies. Such focus on specific dysfunctional attitudes has the potential to increase our knowledge of the occurrence and maintenance of subthreshold depression. For example, perfectionistic dysfunctional attitudes might interact with life events and daily hassles related to achievement (eg, academic burden in college students) to induce subthreshold depression, or such dysfunctional attitudes might generate academic stress, which cause subthreshold depression. Testing these hypotheses will be useful for enhancing our understanding of subthreshold depression. The results of this study provide researchers with the rationale to establish and test such hypotheses.

\section{Conclusion}

A limitation of this study is its cross-sectional design, which makes it impossible to examine the causality between perfectionistic dysfunctional attitudes and subthreshold depression. Based on the findings of this study, however, longitudinal and experimental studies could be designed that do examine causality. The results of this study provide a rationale for conducting such studies in the future. Other limitations are that the sample size was not a priori determined based on a power calculation and the sample size was relatively small, which limits our ability to generalize our findings. However, this study did find evidence of the differential magnitudes of the differences of perfectionistic and dependent dysfunctional attitudes between participants with subthreshold depression and those with no depression. The observed power was sufficient for the test of perfectionistic dysfunctional attitudes.
Based on these findings, it will be possible to conduct further research using an appropriate sample size to examine whether the present findings can be replicated.

\section{Disclosure}

The authors report no conflicts of interest in this work.

\section{References}

1. Bertha EA, Balázs J. Subthreshold depression in adolescence: a systematic review. Eur Child Adolesc Psychiatry. 2013;22(10):589-603.

2. Imamura K, Kawakami N, Furukawa TA, et al. Effects of an Internetbased cognitive behavioral therapy (iCBT) program in Manga format on improving subthreshold depressive symptoms among healthy workers: a randomized controlled trial. PLoS One. 2014;9(5):e97167.

3. Jinnin R, Okamoto Y, Takagaki K, et al. Detailed course of depressive symptoms and risk for developing depression in late adolescents with subthreshold depression: a cohort study. Neuropsychiatr Dis Treat. 2017;13:25-33.

4. Cuijpers P, de Graaf R, van Dorsselaer S. Minor depression: risk profiles, functional disability, health care use and risk of developing major depression. J Affect Disord. 2004;79(1-3):71-79.

5. Takagaki K, Okamoto Y, Jinnin R, et al. Behavioral characteristics of subthreshold depression. J Affect Disord. 2014;168:472-475.

6. Takagaki K, Okamoto Y, Jinnin R et al. Behavioral activation for late adolescents with subthreshold depression: a randomized controlled trial. Eur Child Adolesc Psychiatry. 2016;25(11):1171-1182.

7. Koenig HG. Differences in psychosocial and health correlates of major and minor depression in medically ill older adults. J Am Geriatr Soc. 1997;45(12):1487-1495.

8. Beck AT. Cognitive Therapy and the Emotional Disorders. New York, NY: International Universities Press; 1976.

9. Koenig HG, George LK, Robins CJ, Stangl D, Tweed DL. The development of a Dysfunctional Attitudes Scale for Medically Ill Elders (DASMIE). Clin Gerontol. 1995;15(2):3-22.

10. Eberhart NK, Auerbach RP, Bigda-Peyton J, Abela JRZ. Maladaptive schemas and depression: tests of stress generation and diathesis-stress models. J Soc Clin Psychol. 2011;30(1):75-104.

11. de Graaf LE, Huibers MJ, Cuijpers P, Arntz A. Minor and major depression in the general population: does dysfunctional thinking play a role? Compr Psychiatry. 2010;51(3):266-274.

12. de Graaf LE, Roelofs J, Huibers MJ. Measuring dysfunctional attitudes in the general population: the Dysfunctional Attitude Scale (form A) revised. Cognit Ther Res. 2009;33(4):345-355.

13. Otsubo T, Tanaka K, Koda R, et al. Reliability and validity of Japanese version of the Mini-International Neuropsychiatric Interview. Psychiatry Clin Neurosci. 2005;59(5):517-526.

14. Shima S, Shikano T, Kitamura T, Asai M. New self-rating scales for depression. Clin Psychiat (Tokyo). 1985;27(6):717-723.

15. Tajima M, Akiyama T, Numa H, et al. Reliability and validity of the Japanese version of the 24-item Dysfunctional Attitude Scale. Acta Neuropsychiatr. 2007;19(6):362-367.

16. Cohen J. Statistical power analysis for the behavioral science, 2nd ed. Hillsdale, NJ, USA. Lawrence Erlbaum Associates: 1988.
Psychology Research and Behavior Management

Publish your work in this journal

Psychology Research and Behavior Management is an international, peerreviewed, open access journal focusing on the science of psychology and its application in behavior management to develop improved outcomes in the clinical, educational, sports and business arenas. Specific topics covered in the journal include: Neuroscience, memory and decision making; Behavior

\section{Dovepress}

modification and management; Clinical applications; Business and sports performance management; Social and developmental studies; Animal studies. The manuscript management system is completely online and includes a very quick and fair peer-review system, which is all easy to use. Visit http://www. dovepress.com/testimonials.php to read real quotes from published authors.

Submit your manuscript here: https://www.dovepress.com/psychology-research-and-behavior-management-journal 